\title{
Medium-chain acyl-CoA dehydrogenase deficiency associated with a novel splice mutation in the ACADM gene missed by newborn screening
}

\author{
Sarah C. Grünert ${ }^{1 *+}$, A. Wehrle ${ }^{1 \dagger}$, P. Villavicencio-Lorini ${ }^{1,2}$, E. Lausch¹ ${ }^{1}$ B. Vetter ${ }^{3}$, K. O. Schwab ${ }^{1}$, S. Tucci ${ }^{1}$ \\ and U. Spiekerkoetter ${ }^{1}$
}

\begin{abstract}
Background: Medium-chain acyl-CoA dehydrogenase (MCAD) deficiency is the most common disorder of mitochondrial fatty acid $\beta$-oxidation and a target disease of newborn screening in many countries.
\end{abstract}

Case presentation: We report on two siblings with mild MCAD deficiency associated with a novel splice site mutation in the ACADM gene. The younger sibling was detected by newborn screening, while the older sister was missed, but diagnosed later on by genetic family testing. Both children were found to be compound heterozygous for the common c.985A > G (p.K329E) mutation and a novel splice site mutation, c.600-18G > A, in the ACADM gene. To determine the biological consequence of the c.600-18G > A mutation putative missplicing was investigated at RNA level in granulocytes and monocytes of one of the patients. The splice site mutation was shown to lead to partial missplicing of the ACADM pre-mRNA. Of three detected transcripts two result in truncated, non-functional MCAD proteins as reflected by the reduced octanoyl-CoA oxidation rate in both patients. In one patient a decrease of the octanoyl-CoA oxidation rate was found during a febrile infection indicating that missplicing may be temperature-sensitive.

Conclusions: Our data indicate that the c.600-18G > A variant activates a cryptic splice site, which competes with the natural splice site. Due to only partial missplicing sufficient functional MCAD protein remains to result in mild MCADD that may be missed by newborn screening.

Keywords: ACADM, Missplicing, Newborn screening, False negative

\section{Background}

Medium-chain acyl-CoA dehydrogenase deficiency (MCADD; MIM 201450) is an autosomal recessive disorder of mitochondrial $\beta$-oxidation, caused by mutations in the $A C A D M$ gene. The estimated prevalence of MCADD from newborn screening ranges from 1 in 8,100 to 1 in 27,000 among populations of mostly European descent and is less common in populations of non-European origin [1]. Clinical manifestation comprises hypoketotic hypoglycemia, muscular hypotonia,

\footnotetext{
* Correspondence: sarah.gruenert@uniklinik-freiburg.de

${ }^{\dagger}$ Equal contributors

${ }^{1}$ Center of Pediatrics and Adolescent Medicine, University Hospital Freiburg, Mathildenstraße 1, 79106 Freiburg, Germany

Full list of author information is available at the end of the article
}

lethargy, vomiting, seizures, encephalopathy, coma, and death. Since fatty acid oxidation is essential for energy production during fasting and increased energy expenditure, metabolic decompensations are predominantly precipitated by metabolic stress. In the absence of screening, mortality is up to $26 \%$ [2] and many surviving patients develop severe neurocognitive impairment [1-5]. Since the implementation of tandem mass spectrometry-based newborn screening (NBS) the prognosis of this inherited metabolic disease has significantly improved [6-8]. However, NBS also identifies mild and potentially asymptomatic cases and sometimes also heterozygous individuals, who are at no risk of clinical symptoms.

The typical MCADD acylcarnitine pattern includes elevated levels of C6, C8, C10 and C10:1 acylcarnitines as 
well as increased disease-specific acylcarnitine ratios [C8]/ [C10], [C8]/[C12] and [C8]/[C2] [9]. Additionally, elevated levels of hexanoylglycine, isohexanoylglycine, suberylglycine, and phenylpropionylglycine may be found in the urine of affected individuals. Enzyme activity of MCAD can be determined by measurement of octanoyl-CoA or phenylpropionyl-CoA oxidation rate in lymphocytes and may be used for risk assessment [10]. This is of special importance as the molecular diagnostic after a suggestive NBS result often leads to the identification of novel DNA variants, or "variants of unknown significance" [11].

We report on two asymptomatic siblings with a biochemically mild MCADD phenotype (low levels of MCAD-typical metabolites and high residual enzyme activity). Only one of them was detected by NBS, while the other one was missed. Mutation analysis revealed a known pathogenic missense mutation in combination with a novel splice site mutation in the $A C A D M$ gene. The consequences of this splice site mutation were further characterized.

\section{Patients}

\section{Consent}

All individuals in our study were recruited by physicianinitiated referral. The study was conducted in accordance with the Declaration of Helsinki protocols and approved by the institutional ethics review board of Freiburg University Hospital, Germany. Written informed consent for molecular studies was obtained from the affected individuals and/or their legal guardians in accordance with current German law (GenDG). A copy of the written consent is available for review by the Editor of this journal. Control samples and primary cells were collected from ancestry-, sex- and age-matched healthy individuals under the same criteria and regulations.

\section{Case presentations}

Patient 1, a 7-year-old girl, is the first child of nonconsanguineous German parents. She was born spontaneously at 41 weeks of gestation after an uneventful pregnancy. Birth weight, birth length and head circumference were $3280 \mathrm{~g}$ (56th percentile), $53 \mathrm{~cm}$ (87th percentile) and $35 \mathrm{~cm}$ (50th percentile), respectively. Three hours after birth she was admitted to the intensive care unit with suspected newborn infection. She was pale, tachypnoeic and required CPAP ventilation with up to $60 \%$ oxygen. Laboratory parameters were indicative of systemic infection (Interleukin $6727 \mathrm{pg} /$ $\mathrm{ml}$, reference range $<30 \mathrm{pg} / \mathrm{ml}$ ) and chest X-ray revealed pneumonic infiltrations. Under antibiotic treatment her general condition ameliorated quickly, and the child was dismissed from hospital on day 10. NBS results including acylcarnitine analysis were unremarkable. After dismission from hospital no further problems occurred and the child developed normally.

Five years later her younger sister, patient 2, was born and NBS was suggestive of MCADD. Urinary organic acid analysis biochemically confirmed the diagnosis. Mutation analysis revealed compound heterozygosity for the common missense mutation c.985A $>\mathrm{G}$ and an intronic sequence variant, c.600-18G > A, which has not been described previously. Genetic family screening was performed showing that the older sister (patient 1) was also compound heterozygous for the same two mutations. Heterozygosity for the c.985A > G (p.K329E) mutation was detected in the healthy mother, while the healthy father and the healthy 3-year-old brother were found to be heterozygous for the intronic sequence variant. An overview on the $A C A D M$ genotypes of the two patients, their brother and their parents is shown in Fig. 1.

In the following 3 years acylcarnitine analyses in dried blood spots of both girls were performed repeatedly at

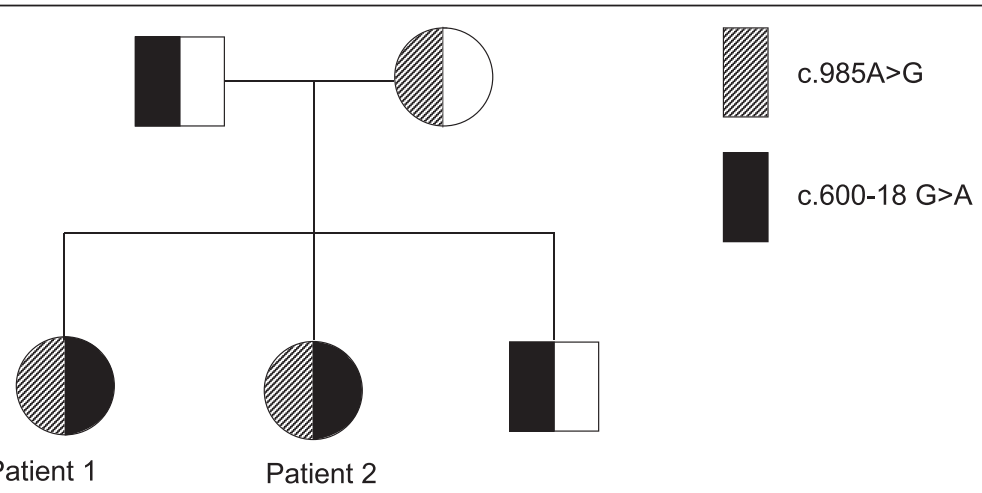

Fig. 1 Pedigree of the patient's family showing haplotypes for the ACADM allele variants in the patient, her siblings and her parents. Males are presented by squares, females by circles. Allele variants without mutation are represented by an open square or circle. Mutated allele variants are indicated by a solid (c.600-18G > A) or a shaded (c.985A > G) square or circle, respectively 
times of physical well-being and yielded variable results (Table 1): In patient 1 three of four samples showed an unremarkable acylcarnitine pattern while patient 2 usually displayed a "typical" MCAD pattern. Free carnitine concentrations were always within the normal range. Analysis of organic acids in urine showed only mildly elevated concentrations of hexanoylglycine and isohexanoylglycine in both patients (Table 1). Suberylglycine was only detected in few samples and the concentration of dicarboxylic acids was usually not elevated. The oxidation rate of octanoyl-CoA was measured in lymphocytes of both patients. In patient 1 enzyme testing was performed during a febrile episode yielding $17 \%$ of healthy controls. In patient 2 , octanoyl-CoA oxidation rate was determined twice, at time of physical wellbeing and during fever showing a residual activity of $24 \%$ and $13 \%$, respectively (Table 1 ).

No inborn errors of metabolism had been detected in other family members so far, and no cases of unexplained death in infancy were reported. When specifically asked, the parents reported that the older sister (patient 1) had well tolerated overnight-fasts of 9-10 h at the age of 9 months. She had had several minor febrile infections including gastroenteritis within her first year of life. At the age of 4.5 years she had suffered from a severe febrile gastroenteritis. During this episode she ate and drank only sparingly for about 4 to 5 days. Despite this catabolic state her vigilance had always been normal with no signs of somnolence or lethargy and clinical symptoms of hypoglycemia were not observed.

\section{Materials and methods}

\section{Oxidation rate of medium-chain acyl-CoAs}

Determination of the MCAD residual activity was achieved by assaying the oxidation of octanoyl-CoA and C10-CoA in lymphocytes by HPLC as described previously [10].

\section{Mutation analysis}

Genomic DNA was extracted from EDTA blood with QIAmp ${ }^{\bullet}$ DNA Mini Kit (Qiagen, Hilden, Germany). All exons and parts of the flanking intronic regions of the $A C A D M$ gene were amplified according to standard protocols using Quick-Load ${ }^{\oplus}$ Taq 2X Master Mix (New England Biolabs, Frankfurt, Germany). Sequencing reactions were performed with the BigDye ${ }^{\otimes}$ Terminator v3.1 Cycle Sequencing Kit (Applied Biosystems, Weiterstadt, Germany) (primer sequences are available on request) and were analysed using a 3730xl DNA Analyzer (Applied Biosystems, USA). Electropherograms were evaluated with the Sequencing Analysis Software v5.4 (Applied Biosystems, USA). To predict a putative missplicing effect of the sequence variant c.600-18G $>$ A the Alamut software 2.3.2 was used.

\section{Definition of RNA splicing patterns}

Peripheral blood samples were fractionated into mononuclear cells and granulocytes using the standard Ficoll protocol. Total RNA was isolated from these cells using TRIzol $^{\circledR}$ Reagent (Life Technologies, USA) followed by a phenol-chloroform extraction, treatment with DNAse I (Roche, Mannheim, Germany) and purification with the RNeasy Mini Kit (Quiagen, Hilden, Germany). Reverse

Table 1 Biochemical data of two siblings with mild MCAD deficiency

\begin{tabular}{|c|c|c|c|c|}
\hline \multirow{2}{*}{\multicolumn{2}{|c|}{ Newborn screening }} & Patient 1 & Patient 2 & Controls \\
\hline & & Unremarkable & Suggestive of MCADD & Unremarkable \\
\hline \multicolumn{5}{|l|}{ Confirmatory testing } \\
\hline \multirow[t]{7}{*}{ Acylcarnitines in dried blood spots (at different time points) } & C6 & $0.06-0.27 \mu \mathrm{mol} / \mathrm{l}$ & $0.13-0.38 \mu \mathrm{mol} / \mathrm{l}$ & $<1 \mu \mathrm{mol} / \mathrm{l}$ \\
\hline & C8 & $0.11-1.56 \mu \mathrm{mol} / \mathrm{l}$ & $0.31-1.5 \mu \mathrm{mol} / \mathrm{l}$ & $<0.4 \mu \mathrm{mol} / \mathrm{l}$ \\
\hline & C10 (including C4:1-DC) & $0.07-0.74 \mu \mathrm{mol} / \mathrm{l}$ & $0.16-0.93 \mu \mathrm{mol} / \mathrm{l}$ & $<0.2 \mu \mathrm{mol} / \mathrm{l}$ \\
\hline & C10:1 & $0.07-0.63 \mu \mathrm{mol} / \mathrm{l}$ & $0.13-0.7 \mu \mathrm{mol} / \mathrm{l}$ & $<0.2 \mu \mathrm{mol} / \mathrm{l}$ \\
\hline & {$[\mathrm{C} 8] /[\mathrm{C} 10]$} & $1.2-2.46$ & $1.62-2.86$ & $<5$ \\
\hline & {$[\mathrm{C} 8] /[\mathrm{C} 12]$} & $4.97-19.3$ & $6.46-17.1$ & $<5$ \\
\hline & {$[\mathrm{C} 8] /[\mathrm{C} 2]$} & $0.01-0.09$ & $0.02-0.4$ & $<0.02$ \\
\hline \multirow[t]{4}{*}{ Urinary organic acids (at different time points) } & Hexanoylglycine & $+/$ n.d. & $+/$ n.d. & n.d. \\
\hline & Isohexanoylglycine & $+/$ n.d. & $+/$ n.d. & n.d. \\
\hline & Suberylglycine & $+/$ n.d. & $+/$ n.d. & n.d. \\
\hline & Dicarboxylic acids & Not elevated & Not elevated & \\
\hline \multirow[t]{2}{*}{ Enzyme activity measurements in lymphocytes } & Octanoyl-CoA-oxidation & $17 \%$ (during fever) & $24 \%$ (without fever) & $100 \%$ \\
\hline & & & $13 \%$ (during fever) & \\
\hline
\end{tabular}


transcription of RNA into cDNA with oligo-dT primers and M-MLV RT (Life Technologies, USA) was conducted according to the manufacturer's protocol. The subsequent PCR amplification was performed with specific primers spanning from exons 7 to 9 of the ACADM gene (For_AACCTGGAGCAGGCTCTGAT and Rev_ACCAGCTCCGTCACCAATTA). PCR products were detected by electrophoresis on a $2 \%$ agarose gel. After excision and gel extraction with the QIAquick Gel Extraction Kit (Qiagen, Hilden, Germany), PCR products were evaluated by sequencing analysis (3130xl Genetic Analyzer, Applied Biosystems, USA). Comparative alignments with the reference coding sequence were carried out by the DNA Sequence Analysis Software Sequencher 4.9 (Gene Codes Corporation, USA).

PCR products of the patient's monocytes cDNA were ligated into the $\mathrm{pCR}^{\mathrm{m}} \mathrm{II}$ Vector and transformed into TOP10F' Chemically Competent E. coli with the TA Cloning ${ }^{\circledR}$ Kit (Life Technologies, USA), according to the manufacturer's protocol. Plasmids containing the different PCR products were purified with the FastPlasmid Mini Kit (5 Prime, Hamburg, Germany). After an EcoRI digestion (New England Biolabs, Frankfurt, Germany) fragments were detected on an agarose gel and different splice variants were identified by their predicted size. To confirm the proportion of the different splicing variants gel fragments were excised, extracted and sequenced as described above.

Fluorescent fragments of DNA were generated via PCR amplification of the cDNA gained from granulocytes and monocytes of the patient and two independent controls with the For_AACCTGGAGCAGGCTCTGAT primer and a Rev_ACCAGCTCCGTCACCAATTA 5' FAM labelled primer, respectively. Fragments were separated using capillary electrophoresis (3130xl Genetic Analyzer, Applied Biosystems, USA) and sized by comparison to a size standard (GeneScan ${ }^{\text {Tx }} 500$ LIZ $^{\circ}$ Size Standard, Life Technologies, USA).

\section{Results}

In the Single Nucleotide Polymorphism database (dbSNP) the sequence variant c.600-18G > A is annotated as an intronic variant with unknown clinical relevance. In silico splice prediction with the Alamut Interactive Biosoftware was performed and all tools integrated in this application (Splice Site Finder (SSF), MaxEntScan (MES), Neural Network (NNSplice) and GeneSplicer) identified the variant as a possible candidate for splicing alteration (Additional file 1: Table S1).

To determine the biological consequence of the c.600-18G > A mutation putative missplicing was investigated at RNA level in granulocytes and monocytes of patient 1. Besides the expected PCR products of ca. $323 \mathrm{bp}$, we observed additional PCR products of 215 and $294 \mathrm{bp}$ which were only present in the patient's sample (Fig. 2). Gel extraction followed by sequencing revealed either a total (215 bp) or partial deletion (294 bp) of exon 8.

In silico translation of both splice variants with the help of the ExPASy translate tool predicts premature stop in the patient's variants due to missplicing and therefore truncated proteins leading most likely to dysfunctional enzyme (Fig. 3).

To quantify missplicing two different approaches were used. In the first approach, cDNA of the patient's monocytes was cloned into the pCRII-TOPO vector. With this method, the different RNA splicing variants could be identified by their calculated transcript lengths. The different splice variants were further confirmed by sequencing. The evaluation of 28 transcripts revealed missplicing in half of all PCR products: Besides the 14 wild type sequences, 5 sequences (18\%) showed skipping of exon 8 , and in 9 sequences (32\%) partial deletion of exon 8 could be confirmed (Additional file 2: Figure S1). Consistent with this finding, the second quantitation approach, based on a fragment analysis with a 5' reverse FAM labelled primer, revealed an approximate ratio of 45:55 of aberrant splicing versus normal

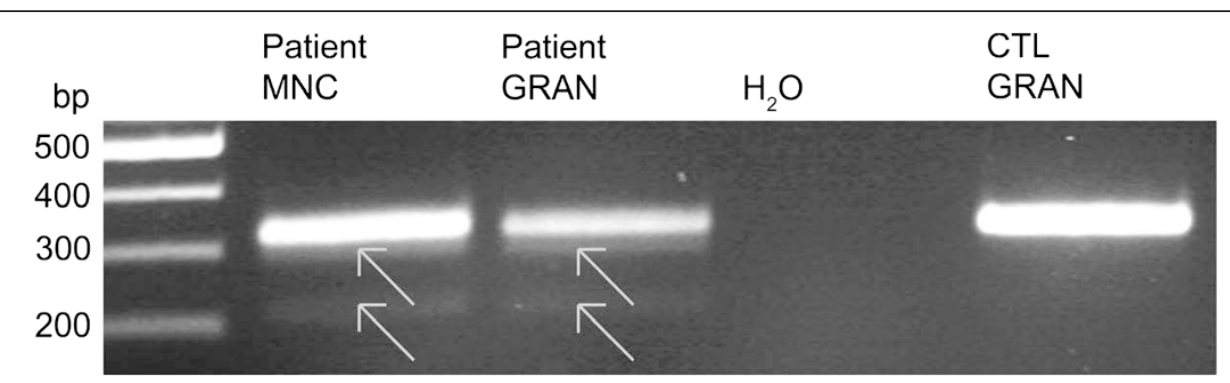

Fig. 2 A novel mutation in the ACADM gene results in aberrant splicing. Gel documentation visualizes the PCR product of a region of 323 bp from exon 7 to 9 of the ACADM gene in CDNA of control granulocytes (CTL GRAN) as well as of monocytes (MNC) and granulocytes (GRAN) of patient 1. Arrows point to the additional PCR products that were detected using CDNA of the patient's granulocytes and monocytes. Analysis of the two additional PCR products in the gel eluates of the patient's samples revealed missplicing resulting in either a partial loss of exon 8 (fragment of 294 bp, upper arrowhead) or complete skipping of exon 8 (fragment of 215 bp, lower arrowhead) 


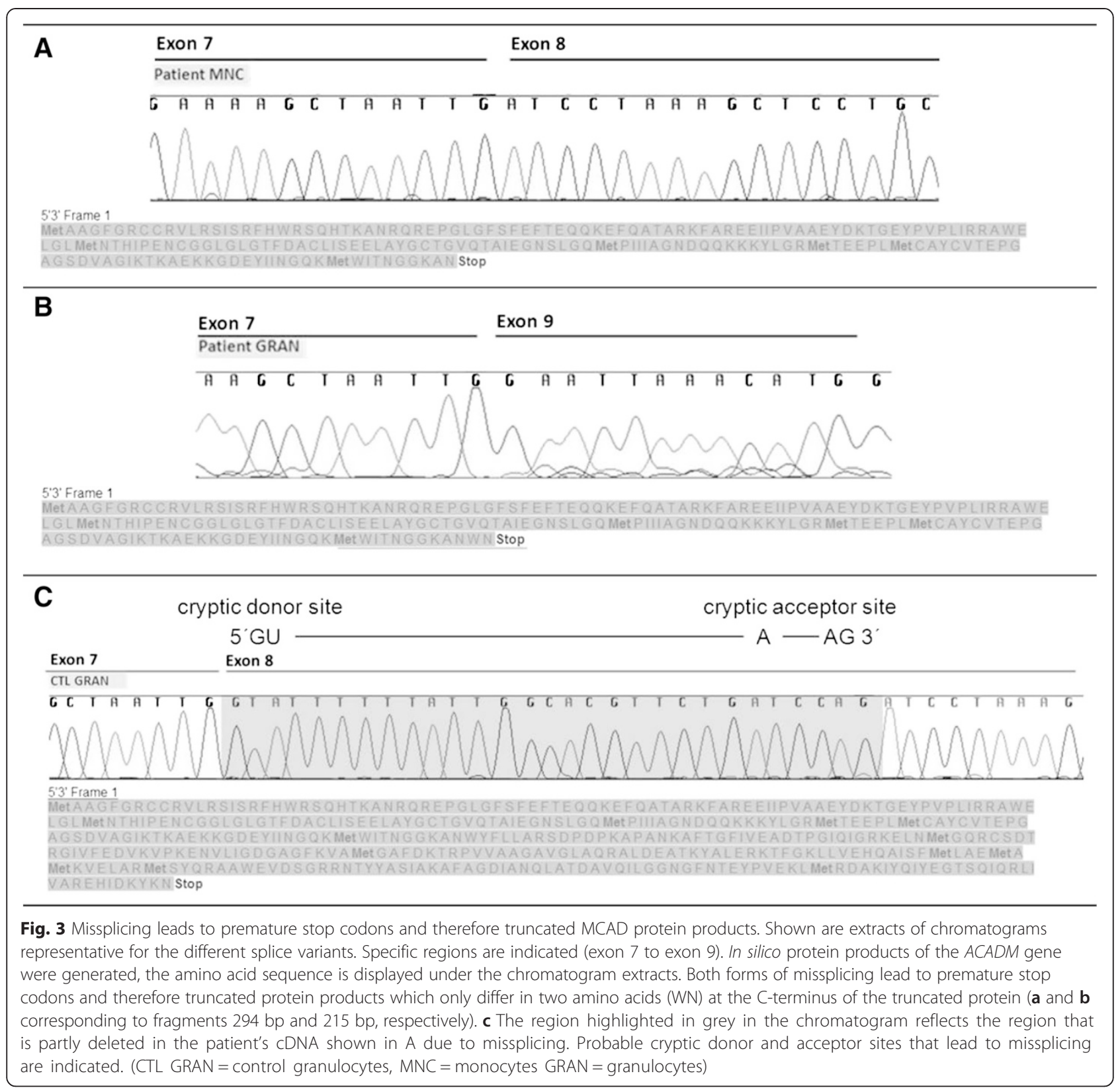

splicing in the patient's monocytes and an approximate ratio of 60:40 in the patient's granulocytes, respectively (Fig. 4, Additional file 3: Table S2). Interestingly, a minor amount ( 10\%) of the transcript with exon 8 skipping could also be detected in the control sample. Therefore, it is conceivable that 'natural' missplicing of the $A C A D M$ RNA also occurs in healthy individuals although to a very limited extent as we could not detect this transcript in the gel.

In a last step, the transcript sequences were scanned for possible cryptic splice sites and a scheme for the aberrant splicing process could be developed. In our model, the c.600-18G > A mutation in intron 7 leads to a splice pattern with strong cryptic donor and acceptor sites that compete with the natural splice sites, resulting either in partial deletion or complete skipping of exon 8 (Fig. 5). We, therefore, presume the sequence variant c.600-18G > A to be likely pathogenic which is also reflected by the reduced octanoyl-CoA oxidation rate in both patients.

\section{Discussion}

We here report on two siblings with mild MCADD, of whom only one was identified by newborn screening. The genotypes of patients identified after implementation of NBS differ from those identified in patients with clinical manifestation $[12,13]$. The mutation c. $985 \mathrm{~A}>\mathrm{G}$ is the 


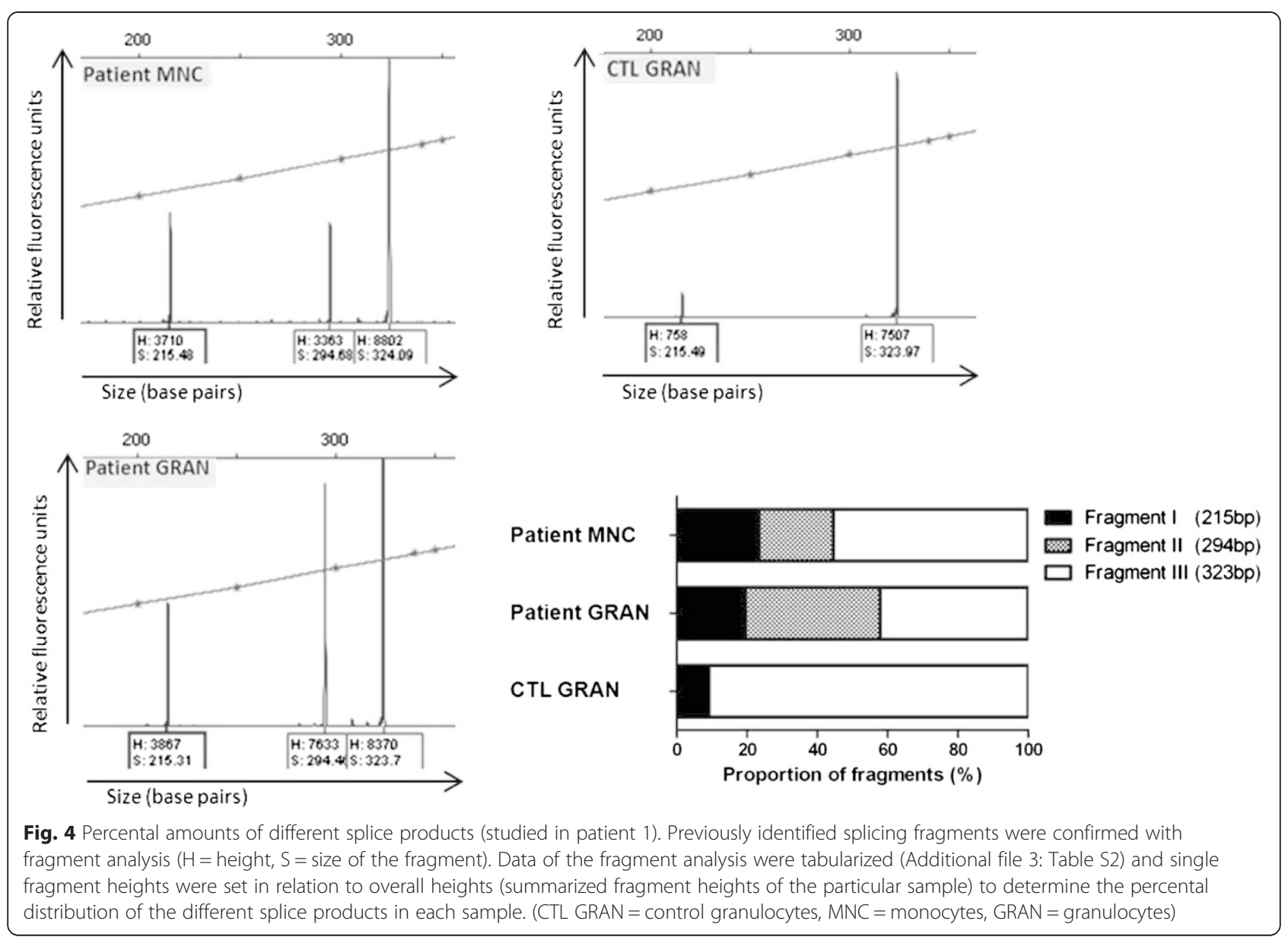

most common mutation in clinically detected patients and has been found in $80 \%$ of these individuals in homozygosity and in $18 \%$ in heterozygosity [14, 15]. This mutation is considerably less frequent in individuals diagnosed by NBS, while numerous novel mutations including a second prevalent mutation, c.199 T > C, have been identified in this group of patients [12, 13]. Individuals carrying these mutations often show significantly lower concentrations of disease-characteristic acylcarnitines $[13,14]$. Our patients were found to be compound heterozygous for the common c.985A > G mutation and a novel splice site mutation, c.600-18G > A. The c.985A $>$ G mutation (K329E) is well characterized with respect to its functional consequences. The MCAD protein is a tetramer of 4 identical subunits. The amino acid change $\mathrm{K} 329 \mathrm{E}$ is located in the $\mathrm{C}$ terminal $\alpha$ domain of the enzyme and compromises tetramer assembly by affecting helix-helix interactions resulting in degradation of the mutant enzyme [16]. The K329E protein variant has been shown to be thermosensitive [17]. Therefore,

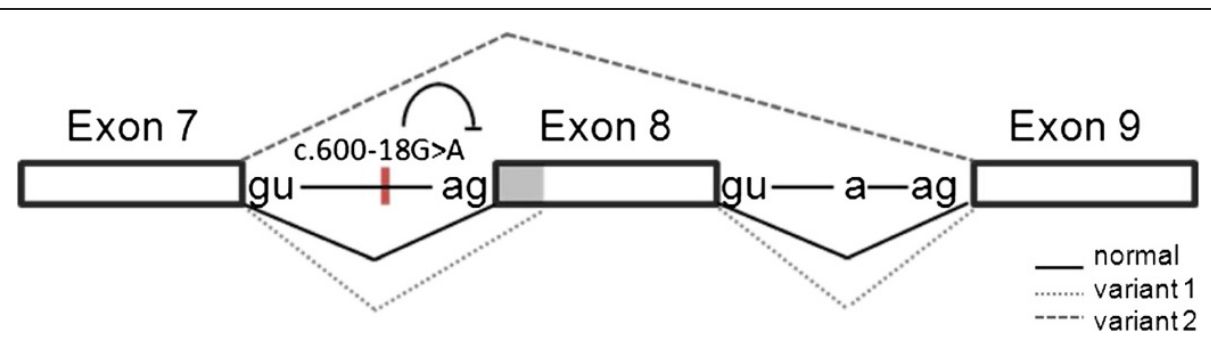

Fig. 5 Working model of the splice reaction with cryptic donor and acceptor sites. Exons are boxed, introns are represented by lines. The mutation in intron 7 of the patient leads to the activation of cryptic donor and acceptor sites that compete with the natural splice site thereby resulting in partial deletion (variant 1) or skipping of exon 8 (variant 2) 
febrile episodes may additionally affect protein stability and function in patients carrying this mutation and may aggravate energy deficiency [18].

The c.600-18G > A mutation has not been described previously. Variant analysis using a prediction software indicated a splice altering mutation. Indeed, we could demonstrate the pathogenicity of this mutation by proof of aberrant splicing. We propose an activation of cryptic splice sites that leads to weakening of the natural splice sites and generates shortened transcripts. Although missplicing is not complete, the combination of both mutations in the compound heterozygous state may account for the reduced enzyme activity and biochemical abnormalities in our patients. The residual enzyme activity measured with octanoyl-CoA as substrate was $13-24 \%$ of healthy controls. These values are higher than those usually found in patients with "classical" MCADD, but lower than those found in heterozygous carriers [19] indicating that the splice site mutation really has a compromising effect on the MCAD protein. Missplicing is known to be highly tissue and cell type specific as can be seen from the different splicing patterns in granulocytes and monocytes of our patients. Though no prediction of the prevalence of missplicing in hepatocytes can be made from our data, it is conceivable that there is a shift in favour of the aberrant splice product in this cell type. Furthermore, it has been reported that splicing processes can be temperature-sensitive. In case that the original splice site is still functional temperature may affect splice site selection in favour of the aberrant splice site $[20,21]$. Interestingly, we found a lower residual activity during a febrile infection than at time of physical well-being in patient 2 (Table 1 ). In the context of MCADD, in which metabolic decompensations are often triggered by febrile infections, this may be of clinical relevance. Enhanced missplicing during episodes of increased energy expenditure may aggravate the cellular energy deficiency and therefore have clinical consequences.

Only one of the two sisters was identified by newborn screening due to a characteristic acylcarnitine pattern. This is notable as it has been shown that even carriers of a single c. $985 \mathrm{~A}>\mathrm{G}$ mutation may display mildly elevated C8 acylcarnitine concentrations [22, 23]. Lehotay et al. reported significantly higher $\mathrm{C} 8$ concentrations of 0.302 $\pm 0.09 \mu \mathrm{mol} / 1$ in c. $985 \mathrm{~A}>\mathrm{G}$ heterozygotes compared to healthy controls, while the $\mathrm{C} 8$ concentration in our compound heterozygous patient $(0.17 \mu \mathrm{mol} / \mathrm{l})$ was even below this range. High $\mathrm{C} 8$ levels have been shown to be associated with severe mutations including the common c.985G > C mutation (in homozygosity), deletions, nonsense or splice site mutations [24]. In a study with 34 MCAD deficient individuals evaluating the correlation between genotype and biochemical phenotype, patients homozygous for c.985A $>\mathrm{G}$ had the highest levels of neonatal octanoylcarnitine followed by compound heterozygotes of c.985A $>\mathrm{G}$ and other mutations. Patients without c. $985 \mathrm{~A}>\mathrm{G}$ and compound heterozygotes for c. $985 \mathrm{~A}>\mathrm{G}$ and c.199 $\mathrm{T}>\mathrm{C}$ had the lowest levels of C8. According to this, an intermediate elevation of the $\mathrm{C} 8$ acylcarnitine concentration would have been expected in our patient.

Even if the high residual MCAD activity and the mild biochemical expression in our patients are suggestive of a presumably low clinical penetrance, the potential risk of metabolic decompensation during severe catabolism cannot be estimated and the clinical relevance of mild MCADD is difficult to establish. Although the majority of symptomatic patients present within the first three years of life, several late onset cases have been reported [25, 26]. Both girls have remained asymptomatic so far, however, they are only 3 and 7 years old, respectively. Nevertheless, it is of great interest that patient 1 has experienced many infections and catabolic conditions before diagnosis by family screening not resulting in any clinical symptoms. The natural course of the disease in individuals with novel mutations is generally unknown [16]. Many of these children may remain asymptomatic even without detection by NBS. This is reflected by the much higher detection rate of MCADD by NBS compared to clinically detected cases in unscreened populations. In contrast, one more recent study on structural alterations of several mutant variants proposed that novel mutations found in NBS, including the c.199 $\mathrm{T}>\mathrm{C}$ mutation, do not bear a lower risk of metabolic decompensation than that associated with mutations detected in clinically ascertained patients [16], although, to our knowledge, only one individual carrying the c.199 $\mathrm{T}>\mathrm{C}$ mutation together with a second mutation has ever been symptomatic during follow-up. The mother sought emergency room care for the infant two times in the first year for listlessness associated with gastroenteritis. At age 22 months altered mental status and variable hypo- and hyperglycemia were reported during hospital admission for hand/foot/mouth disease [24]. Residual enzyme activities in patients with proposed mild MCADD are in some cases in the same activity range as in definite heterozygous parents questioning the clinical relevance of the mutations [10]. So far, there is no consensus to consider certain $A C A D M$ genotypes as safe and protective from clinical symptoms [16, 24], however, we are also aware of an overtreatment of many individuals identified by newborn screening. The same uncertainty is true with respect to the interpretation of the biochemical phenotype despite a strong relationship between initial C8 levels and outcome [24]. 


\section{Conclusion}

The novel splice variant c.600-18G > A was shown to activate a cryptic splice site, which competes with the natural splice site. Due to only partial missplicing sufficient functional MCAD protein remains to result in clinically and biochemically mild MCADD that may be missed by newborn screening. If patients with such mild phenotypes are at risk of fatal metabolic decompensations remains a matter of debate.

\section{Additional files}

Additional file 1: Table S1. In silico splice prediction of the Alamut Interactive Biosoftware. Note that all tools integrated in this application (Splice Site Finder (SSF), MaxEntScan (MES), Neural Network (NNSplice) and GeneSplicer) identified the variant as a possible candidate for splicing alteration.

Additional file 2: Figure S1. Gel documentation of PCR products of the patient's monocyte cDNA after EcoRI digestion. Fragments were detected on an agarose gel. Splice variants were identified by their predicted size and confirmed by sequence analysis. Missplicing was detected in half of the fragments.

Additional file 3: Table S2. Tabularized data of the fragment analysis. Different fragments are indicated with I (215 bp), II (294 bp) or III (323 bp). Single fragment heights were set in relation to overall heights (summarized fragment heights of the particular sample) to determine the percental distribution of the different splice products in each sample. CTL GRAN = control garanulocytes, Patient GRAN = granulocytes of patient, Patient MNC = monocytes of patients.

\section{Abbreviations}

C8: Octanoyl-carnitine; MS/MS: Tandem mass spectrometry; MCAD: Medium chain acyl-CoA dehydrogenase; MCADD: Medium chain acyl-CoA dehydrogenase deficiency; NBS: Newborn screening.

\section{Competing interests}

The authors declare that they have no competing interests.

\section{Authors' contributions}

SG collected clinical data and laboratory samples of the patients and drafted the manuscript. AW, PV-L and EL performed the molecular studies and characterization of the novel splice site mutation and critically revised the manuscript. BV and KOS contributed to data collection. ST perfomed the enzymatic investigations. All authors participated in data discussion and critically revised the manuscript. All authors read and approved the final manuscript.

\section{Acknowledgement}

The authors are thankful to the family for their support and interest in this study.

\section{Author details}

${ }^{1}$ Center of Pediatrics and Adolescent Medicine, University Hospital Freiburg, Mathildenstraße 1, 79106 Freiburg, Germany. ${ }^{2}$ Present address: Department of Human Genetics, Halle University Hospital, Ernst-Grube-Str. 30, 06097 Halle, Germany. ${ }^{3}$ Römerstrasse 38, 79423 Heitersheim, Germany.

Received: 7 October 2014 Accepted: 9 July 2015

Published online: 30 July 2015

\section{References}

1. Grosse SD, Khoury MJ, Greene CL, Crider KS, Pollitt RJ. The epidemiology of medium chain acyl-CoA dehydrogenase deficiency: an update. Genet Med. 2006;8(4):205-12

2. Touma EH, Charpentier C. Medium chain acyl-CoA dehydrogenase deficiency. Arch Dis Child. 1992;67(1):142-5.
3. Iafolla AK, Thompson Jr RJ, Roe CR. Medium-chain acyl-coenzyme A dehydrogenase deficiency: clinical course in 120 affected children. J Pediatr. 1994;124(3):409-15.

4. Pollitt RJ, Leonard JV. Prospective surveillance study of medium chain acyl-CoA dehydrogenase deficiency in the UK. Arch Dis Child. 1998;79(2):116-9.

5. Wilcken B, Hammond J, Silink M. Morbidity and mortality in medium chain acyl coenzyme A dehydrogenase deficiency. Arch Dis Child. 1994;70(5):410-2.

6. Nennstiel-Ratzel U, Arenz S, Maier EM, Knerr I, Baumkotter J, Roschinger W, et al. Reduced incidence of severe metabolic crisis or death in children with medium chain acyl-CoA dehydrogenase deficiency homozygous for c.985A > G identified by neonatal screening. Mol Genet Metab. 2005;85(2):157-9.

7. Wilcken B. Fatty acid oxidation disorders: outcome and long-term prognosis. J Inherit Metab Dis. 2010;33(5):501-6.

8. Wilcken B, Haas M, Joy P, Wiley V, Chaplin M, Black C, et al. Outcome of neonatal screening for medium-chain acyl-CoA dehydrogenase deficiency in Australia: a cohort study. Lancet. 2007;369(9555):37-42.

9. Millington DS, Roe CR. Medium-chain acyl-CoA dehydrogenase deficiency. N Engl J Med. 1989;320(18):1219.

10. Sturm M, Herebian D, Mueller M, Laryea MD, Spiekerkoetter U. Functional effects of different medium-chain acyl-CoA dehydrogenase genotypes and identification of asymptomatic variants. PLoS One. 2012;7(9):e45110.

11. Smith EH, Thomas C, McHugh D, Gavrilov D, Raymond K, Rinaldo P, et al. Allelic diversity in MCAD deficiency: the biochemical classification of 54 variants identified during 5 years of ACADM sequencing. Mol Genet Metab. 2010;100(3):241-50.

12. Andresen BS, Dobrowolski SF, O'Reilly L, Muenzer J, McCandless SE, Frazier DM, et al. Medium-chain acyl-CoA dehydrogenase (MCAD) mutations identified by MS/MS-based prospective screening of newborns differ from those observed in patients with clinical symptoms: identification and characterization of a new, prevalent mutation that results in mild MCAD deficiency. Am J Hum Genet. 2001;68(6):1408-18.

13. Maier EM, Liebl B, Roschinger W, Nennstiel-Ratzel U, Fingerhut R, Olgemoller $B$, et al. Population spectrum of ACADM genotypes correlated to biochemical phenotypes in newborn screening for medium-chain acyl-CoA dehydrogenase deficiency. Hum Mutat. 2005;25(5):443-52.

14. Waddell L, Wiley V, Carpenter K, Bennetts B, Angel L, Andresen BS, et al. Medium-chain acyl-CoA dehydrogenase deficiency: genotype-biochemical phenotype correlations. Mol Genet Metab. 2006;87(1):32-9.

15. Yokota I, Coates PM, Hale DE, Rinaldo P, Tanaka K. Molecular survey of a prevalent mutation, 985A-to-G transition, and identification of five infrequent mutations in the medium-chain Acyl-CoA dehydrogenase (MCAD) gene in 55 patients with MCAD deficiency. Am J Hum Genet. 1991;49(6):1280-91.

16. Maier EM, Gersting SW, Kemter KF, Jank JM, Reindl M, Messing DD, et al. Protein misfolding is the molecular mechanism underlying MCADD identified in newborn screening. Hum Mol Genet. 2009;18(9):1612-23.

17. Bross $P$, Jespersen $C$, Jensen $T G$, Andresen BS, Kristensen MJ, Winter $V$, et al. Effects of two mutations detected in medium chain acyl-CoA dehydrogenase (MCAD)-deficient patients on folding, oligomer assembly, and stability of MCAD enzyme. J Biol Chem. 1995;270(17):10284-90.

18. Dessein AF, Fontaine M, Andresen BS, Gregersen N, Brivet M, Rabier D, et al. A novel mutation of the ACADM gene (c.145C > G) associated with the common c.985A > G mutation on the other ACADM allele causes mild MCAD deficiency: a case report. Orphanet J Rare Di. 2010;5:26.

19. Zschocke J, Schulze A, Lindner M, Fiesel S, Olgemoller K, Hoffmann GF, et al. Molecular and functional characterisation of mild MCAD deficiency. Hum Genet. 2001;108(5):404-8.

20. Gemignani F, Sazani P, Morcos P, Kole R. Temperature-dependent splicing of beta-globin pre-mRNA. Nucleic Acids Res. 2002;30(21):4592-8.

21. Wu Y, Kuivaniemi H, Tromp G, Strobel D, Romanic AM, Prockop DJ. Temperature sensitivity of aberrant RNA splicing with a mutation in the $\mathrm{G}+5$ position of intron 37 of the gene for type III procollagen from a patient with Ehlers-Danlos syndrome type IV. Hum Mutat. 1993;2(1):28-36.

22. Blois B, Riddell C, Dooley K, Dyack S. Newborns with C8-acylcarnitine level over the 90th centile have an increased frequency of the common MCAD 985A > G mutation. J Inherit Metab Dis. 2005;28(4):551-6.

23. Lehotay DC, LePage J, Thompson JR, Rockman-Greenberg C. Blood acylcarnitine levels in normal newborns and heterozygotes for 
medium-chain acyl-CoA dehydrogenase deficiency: a relationship between genotype and biochemical phenotype? J Inherit Metab Dis. 2004;27(1):81-8.

24. Arnold GL, Saavedra-Matiz CA, Galvin-Parton PA, Erbe R, Devincentis E, Kronn $D$, et al. Lack of genotype-phenotype correlations and outcome in MCAD deficiency diagnosed by newborn screening in New York State. Mol Genet Metab. 2010;99(3):263-8

25. Raymond K, Bale AE, Barnes CA, Rinaldo P. Medium-chain acyl-CoA dehydrogenase deficiency: sudden and unexpected death of a 45 year old woman. Genet Med. 1999;1(6):293-4.

26. Wilhelm GW. Sudden death in a young woman from medium chain acyl-coenzyme A dehydrogenase (MCAD) deficiency. J Emerg Med. 2006:30(3):291-4.

\section{Submit your next manuscript to BioMed Central and take full advantage of:}

- Convenient online submission

- Thorough peer review

- No space constraints or color figure charges

- Immediate publication on acceptance

- Inclusion in PubMed, CAS, Scopus and Google Scholar

- Research which is freely available for redistribution 